\title{
The Role of Endogenous Reactive Oxygen Species in Cardiac Myocyte Autophagy
}

\author{
Jia-Pu WANG ${ }^{1,2,3}$, Rui-Fang CHI ${ }^{1,2,3}$, Jia LIU $^{2,3}$, Yong-Zhi DENG ${ }^{2,3}$, Xue-Bin HAN ${ }^{2,3}$, \\ Fu-Zhong QIN ${ }^{1,2,3}$, Bao LI ${ }^{1,4}$
}

${ }^{1}$ Shanxi Medical University, Taiyuan, Shanxi, China, ${ }^{2}$ The Affiliated Cardiovascular Hospital of Shanxi Medical University, Taiyuan, Shanxi, China, ${ }^{3}$ Shanxi Province Cardiovascular Hospital, Taiyuan, Shanxi, China, ${ }^{4}$ The Second Hospital of Shanxi Medical University, Taiyuan, Shanxi, China

Received April 15, 2017

Accepted August 8, 2017

On-line November 10, 2017

\section{Summary}

Autophagy is implicated in the maintenance of cardiac homeostasis. Autophagy is activated in heart failure, in which reactive oxygen species (ROS) are increased. Exogenous ROS have been shown to induce cardiomyocyte autophagy alterations. However, little is known about the influences of physiological levels of endogenous ROS on cardiomyocyte autophagy. In the present study, we tested the hypothesis that endogenous ROS in cardiomyocytes play an important role in inducing autophagy. Cultured $\mathrm{H} 9 \mathrm{C} 2$ cardiomyocytes or Sprague-Dawley rats were treated with the antioxidant $\mathrm{N}$-acetyl-cysteine (NAC) or the superoxide dismutase mimic tempol under the basal or nutrient deprivation conditions. The autophagic flux was assessed by the lysosomal inhibitor chloroquine. In $\mathrm{H} 9 \mathrm{C} 2$ cardiomyocytes, under a basal condition, NAC or tempol increased the ratio of LC3 II/I proteins and reduced LC3 II autophagic flux. Under nutrient deprivation, NAC increased the LC3 II/I ratio and reduced LC3 II autophagic flux. In vivo studies in rats, NAC treatment increased the LC3 II/I ratio and p-Akt protein expression in myocardium. We concluded that the antioxidants reduced autophagic flux in cardiomyocytes under the basal or nutrient deprivation conditions, suggesting that endogenous ROS promote autophagy flux under physiological conditions, and this effect is mediated, at least in part, through Akt inhibition.

\section{Key words}

Reactive oxygen species - Endogenous - Autophagy • Cardiomyocytes • Rats

\section{Corresponding author}

F.-Z. Qin, Shanxi Medical University, 56 South Xinjian Road, Taiyuan 030001, Shanxi, China; The Affiliated Cardiovascular Hospital of Shanxi Medical University, 18 Yifen Street, Taiyuan 030024, Shanxi, China. E-mail: sxcvh13@sina.com

\section{Introduction}

Autophagy is a degradation process in which cytosolic proteins and organelles are degraded and recycled through lysosomes (Lavandero et al. 2013). It occurs in the normal myocardium and plays an important role in maintaining cardiac structure and function. Inefficient autophagy or its absence causes cardiac dysfunction and dilation (Nakai et al. 2007). Increasing evidence suggests that autophagy is activated during various pathologic conditions in the heart. For example, myocyte autophagy is activated during acute myocardial ischemia, myocardial ischemia and reperfusion (Matsui et al. 2007), myocardial infarction (Kanamori et al. 2011), cardiac hypertrophy and heart failure (Zhu et al. 2007). The function of autophagy in the heart appears to be stimulus dependent.

Several mechanisms mediate myocyte autophagy. One of these mechanisms is the production of reactive oxygen species (ROS). Antioxidant N-2-mercaptopropionyl glycine attenuates myocyte autophagy during myocardial ischemia and reperfusion, suggesting oxidative stress mediates myocyte autophagy 
(Hariharan et al. 2011). In cardiac myocytes in vitro, glucose deprivation-stimulated ROS induce autophagy (Marambio et al. 2010). However, little is known about the influences of normal physiological levels of endogenous ROS on cardiac myocyte autophagy. We hypothesize that physiological levels of ROS production in cardiac myocytes play an important role in inducing autophagy. In the present study, we first evaluate the functional roles of endogenous ROS in the regulation of basal and moderate stress-triggered autophagy in cardiomyocytes.

The sources of ROS include mitochondrial respiration, NADPH oxidase and xanthine oxidase (Santos et al. 2016). NADPH oxidase is the major source of ROS in cardiomyocytes. However, the contribution of the NADPH oxidase-derived ROS to the regulation of basal autophagy in cardiac myocytes remains to be fully elucidated. Therefore, the second objective of this study is to evaluate the functional roles of NADPH oxidasederived ROS in the regulation of basal autophagy in cardiomyocytes.

The mechanisms through which ROS regulate autophagy have not been fully understood. High levels of ROS during myocardial ischemia and reperfusion induce myocyte autophagy mediated by Beclin1 expression (Hariharan et al. 2011). A high dose of hydrogen peroxide $\left(\mathrm{H}_{2} \mathrm{O}_{2}\right)$ induces myocyte autophagy in association with decreased phosphorylated mTOR protein expression and increased phosphorylated AMPK expression (Essick et al. 2013). However, it is unknown about the mechanisms of endogenous ROS-regulated myocyte autophagy. Accordingly, the third objective of this study is to determine the signaling transduction pathways of physiological levels of ROS-regulated myocyte autophagy.

\section{Methods}

\section{H9C2 cardiomyocyte culture}

The H9C2 cardiomyocytes were cultured in $75 \mathrm{~cm}^{2}$ flask in Dulbecco's modified Earle's medium (DMEM) (Gibco, Life Technologies, USA) containing $4.5 \mathrm{~g} / \mathrm{l}$ D-glucose, $3.7 \mathrm{~g} / \mathrm{l}$ sodium bicarbonate and $110 \mathrm{mg} / \mathrm{l}$ sodium pyruvate, supplemented with $10 \%$ fetal bovine serum (Australia) and penicillin (100 units/ml) and streptomycin $(100 \mu \mathrm{g} / \mathrm{ml})$ in a humidified incubator with $95 \%$ air and $5 \% \mathrm{CO}_{2}$ at $37{ }^{\circ} \mathrm{C}$, as described in our previous study ( $\mathrm{Li}$ et al. 2015). After 4 to 5 days, cells were passaged at a 1:5 ratio and seeded at the density of
$0.15 \times 10^{6}$ cells per $35-\mathrm{mm}$ well of six-well plates. These cells were cultured for 4 days and then performed treatments.

\section{$\mathrm{H}_{2} \mathrm{O}_{2}$ treatment}

H9C2 cardiomyocytes were exposed to 10, 100, and $1,000 \mu \mathrm{M}$ of $\mathrm{H}_{2} \mathrm{O}_{2}$ (Sigma-Aldrich, St. Louis, MO, USA) for $60 \mathrm{~min}$ or phosphate-buffered saline (PBS) containing $0.1 \%$ bovine serum albumin used as a control.

\section{Antioxidant treatment}

H9C2 cardiomyocytes were incubated with N-acetyl-cysteine (NAC) (Sigma-Aldrich, St. Louis, MO, USA) at $2 \mathrm{mM}$ or tempol (Sigma-Aldrich, St. Louis, MO, USA) at $10 \mathrm{mM}$ for $6 \mathrm{~h}$. PBS containing $0.1 \%$ bovine serum albumin was used as a control in all protocols. The dosage for NAC and tempol used in this study was based on the previous studies (Peng et al. 2011, Rahman et al. 2014). NAC is a precursor of the cellular antioxidant glutathione. Tempol is a membrane-permeable piperidine nitroxide that mimics SODs by dismuting $\mathrm{O}_{2}^{-}$anions. Tempol also detoxifies redox-reactive transition metal ions and directly reacts with many ROS forming adducts.

\section{NADPH oxidase inhibitor apocynin treatment}

H9C2 cardiomyocytes were treated with apocynin $(100 \mu \mathrm{mol} / \mathrm{l})$ (Sigma-Aldrich, St. Louis, MO, USA) for $6 \mathrm{~h}$. DMSO $(0.02 \%)$ was used as control. The dose used in this study was based on the previous report (Qin et al. 2007).

\section{Nutrient deprivation for induction of autophagy}

H9C2 cardiomyocytes were cultured in glucoseand serum-free DMEM for $6 \mathrm{~h}$ (a nutrient deprivation condition) for induction of autophagy.

\section{Autophagic flux measurements in vitro}

H9C2 cardiomyocytes were incubated in DMEM containing vehicle (PBS), NAC, tempol, PBS plus a lysosomal inhibitor chloroquine, NAC plus chloroquine or tempol plus chloroquine for $6 \mathrm{~h}$ (a basal condition) or glucose-and serum-free DMEM containing vehicle (PBS), NAC, tempol, PBS plus chloroquine, NAC plus chloroquine or tempol plus chloroquine for $6 \mathrm{~h}$ (a nutrient deprivation condition). LC3 BII protein detection was performed using Western blot. The autophagic flux was assessed by the lysosomal inhibitor chloroquine (Rahman et al. 2014), and determined by calculating the difference between absolute LC3 BII protein levels measured in the 
presence and absence of chloroquine in $\mathrm{H} 9 \mathrm{C} 2$ cardiomyocytes preincubated with vehicle, NAC or tempol.

\section{Effects of antioxidants on myocyte autophagy in vivo}

The protocol for the care and use of all animals in this study was approved by the Shanxi Medical University Committee on Animal Resources and conformed to "the guiding principles in the Care and Use of Animals" of the American Physiological Society and the Guide for the Care and Use of Laboratory Animals as outlined by the National Research Council and the US National Institutes of Health Publication No. 85-23, revised 1996. Male Sprague-Dawley rats weighing 180-200 g were obtained from the Academy of Military Medical Sciences. All animals had access to the standard laboratory diet and drinking water. The animals were randomized to receive an antioxidant NAC (Sigma-Aldrich, St Louis, MO, USA) $(500 \mathrm{mg} / \mathrm{kg} /$ day) or normal saline treatment for 2 weeks $(n=6)$. The dose used in this study was based on the previous reports (Grieve et al. 2006).

\section{Western blot}

H9C2 cardiomyocytes or left ventricular myocardial tissue were homogenized in a lysis buffer containing $1 \mathrm{M}$ Tris- $\mathrm{HCl}(\mathrm{pH} 7.4), 0.25 \mathrm{M}$ sucrose, $0.5 \mathrm{M}$ EDTA (pH 8.0), $100 \mathrm{mM}$ dithiothreitol, $100 \mathrm{mM}$ phenylmethylsulfonyl fluoride, aprotinin $(2.2 \mathrm{mg} / \mathrm{ml})$, leupeptin $(5 \mathrm{mg} / \mathrm{ml})$. The homogenate was centrifuged at $12,000 \times \mathrm{g}$ for $30 \mathrm{~min}$ at $4{ }^{\circ} \mathrm{C}$ and the supernatant was collected as protein extracts. Protein extracts $(40-60 \mu \mathrm{g})$ were resolved by $12 \%$ SDS-polyacrylamide gel and transferred to polyvinylidene fluoride membranes. The blots were incubated overnight with rabbit anti-LC3 (dilution 1:500) (Novus, Biologicals, Littleton, CO, USA), autophagy-related gene 5 (Atg5) (dilution 1:100), sequestosome 1 (SQSTM1/P62) (dilution 1:1,000), adenosine 5'-monophosphate (AMP)-activated protein kinase (AMPK) (dilution 1:1,000) and protein kinase B (Akt, a serine/threonine-specific protein kinase) (dilution 1:200) (Santa Cruz Biotechnology, Dallas, TX, USA) polyclonal antibodies. Mouse anti-GAPDH monoclonal antibody (dilution 1:10,000) (Abcam, Cambridge, MA, USA) was used to confirm equal loading conditions. The blots were then incubated with the secondary antibodies goat anti-rabbit IgG-HRP or goat anti-mouse IgG-HRP (dilution 1:2,000). The Western blot bands were analyzed using the Image Lab ${ }^{\mathrm{TM}}$ acquisition and analysis software (Bio-Lab, Hercules, CA, USA). The optical density of tissue samples was normalized to a control sample in an arbitrary densitometry unit.

\section{Statistical analysis}

Data are presented as mean \pm SEM. Student's t-test for unpaired data was used to determine the statistical significance of differences between the two means. The statistical significance of differences among groups was determined using analysis of variance and the Bonferroni correction for multiple comparisons. A value of $\mathrm{P}<0.05$ was considered statistically significant.

\section{Results}

The effects of exogenous $\mathrm{H}_{2} \mathrm{O}_{2}$ on autophagy in $\mathrm{H} 9 \mathrm{C} 2$ cardiomyocytes

Evidence has accumulated that autophagy is activated under pathological conditions in the heart (Hariharan et al. 2011). For example, autophagy is activated during myocardial ischemia and reperfusion and heart failure (Nakai et al. 2007, Zhu et al. 2007), which are associated with increased oxidative stress (Burgoyne et al. 2012). To determine whether increased oxidative stress regulates myocyte autophagy, $\mathrm{H} 9 \mathrm{C} 2$ cardiomyocytes were treated with exogenous $\mathrm{H}_{2} \mathrm{O}_{2}(10$, 100 or $1,000 \mu \mathrm{M})$ for $60 \mathrm{~min}$. Figures $1 \mathrm{~A}$ and $1 \mathrm{~B}$ show that $\mathrm{H}_{2} \mathrm{O}_{2}$ at $10 \mu \mathrm{M}$ had no effect on the ratio of LC3 II to LC3 I proteins, a marker of autophagy, in $\mathrm{H} 9 \mathrm{C} 2$ cardiomyocytes, but $\mathrm{H}_{2} \mathrm{O}_{2}$ at 100 or $1,000 \mu \mathrm{M}$ caused a marked increase in autophagy in $\mathrm{H} 9 \mathrm{C} 2$ cardiomyocytes. Figures 1C and 1D show that the autophagic protein Atg5 expression had no change in $\mathrm{H} 9 \mathrm{C} 2$ cardiomyocytes after exposure to $10 \mu \mathrm{M}$ of $\mathrm{H}_{2} \mathrm{O}_{2}$, but $\mathrm{Atg} 5$ protein expression was significantly increased in $\mathrm{H} 9 \mathrm{C} 2$ cardiomyocytes after exposure to 100 or $1,000 \mu \mathrm{M}$ of $\mathrm{H}_{2} \mathrm{O}_{2}$. These findings suggest that high concentrations of exogenous $\mathrm{H}_{2} \mathrm{O}_{2}$ significantly induce myocyte autophagy.

The role of endogenous ROS in autophagy in $\mathrm{H} 9 \mathrm{C} 2$ cardiomyocytes

To determine the functional roles of endogenous ROS in the regulation of myocyte autophagy, two general antioxidants, NAC and tempol, were used to treat $\mathrm{H} 9 \mathrm{C} 2$ cardiomyocytes. Under a basal condition, $\mathrm{H} 9 \mathrm{C} 2$ cardiomyocytes were treated with NAC, tempol or placebo for $6 \mathrm{~h}$. Figures $2 \mathrm{~A}$ and $2 \mathrm{~B}$ show that NAC induced a significant increase in the LC3 II/I ratio. Similarly, tempol also caused an increase in the LC3 II/I ratio (Figs 2C, 2D). These findings suggest that NAC or 
tempol triggers the accumulation of autophagosomes, which is the result of either increased synthesis or decreased degradation.

To determine whether NADPH oxidase is a major source of endogenous ROS which regulate autophagy, H9C2 cardiomyocytes were treated with apocynin, an inhibitor of NADPH oxidase or placebo for $6 \mathrm{~h}$. Figures $2 \mathrm{E}$ and $2 \mathrm{~F}$ show that apocynin markly increased the LC3 II/I ratio. These results suggest that NADPH oxidase may be a major source of endogenous ROS which regulate myocyte autophagy.
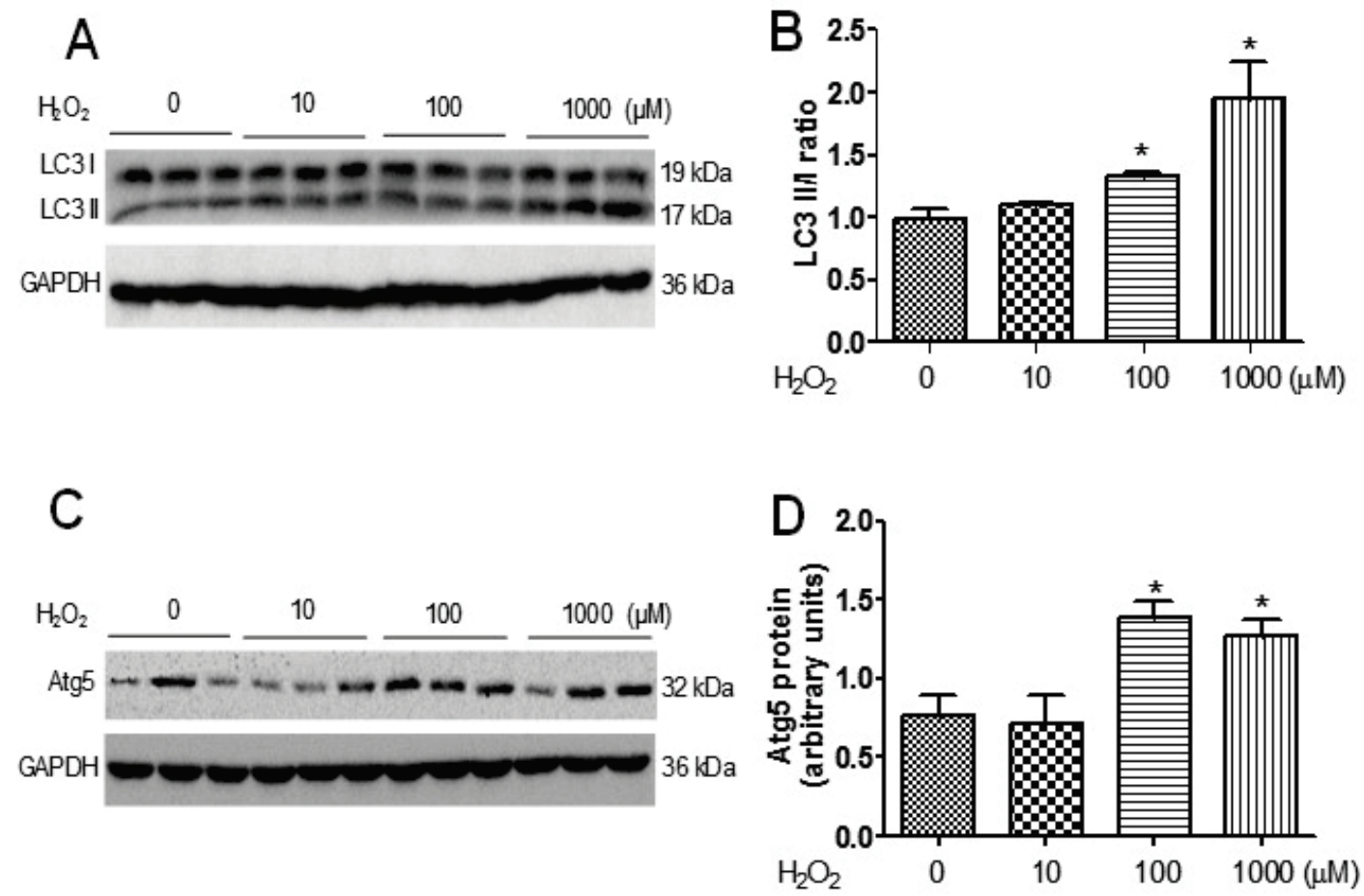

Fig. 1. Effects of exogenous hydrogen peroxide $\left(\mathrm{H}_{2} \mathrm{O}_{2}\right)$ on autophagy in $\mathrm{H} 9 \mathrm{C} 2$ cardiomyocytes. (A) Representative Western blots of LC3 II and I proteins in $\mathrm{H} 9 \mathrm{C} 2$ cardiomyocytes exposed to 10,100 and $1,000 \mu \mathrm{M}$ of $\mathrm{H}_{2} \mathrm{O}_{2}$ or phosphate-buffered saline (PBS) containing $0.1 \%$ bovine serum albumin (BSA) as a control for $1 \mathrm{~h}$. Equal loading of proteins is illustrated by GAPDH bands. (B) Respective group densitometry analysis. Values are means $\pm \mathrm{SEM} ; \mathrm{n}=6$. $* \mathrm{P}<0.01$ vs. controls. (C) Representative Western blots of Atg5 protein in $\mathrm{H} 9 \mathrm{C} 2$ cardiomyocytes exposed to 10,100 and $1,000 \mu \mathrm{M}$ of $\mathrm{H}_{2} \mathrm{O}_{2}$ or PBS containing $0.1 \%$ BSA as a control for $1 \mathrm{~h}$. Equal loading of proteins is illustrated by GAPDH bands. (D) Respective group densitometry analysis. Values are means $\pm \mathrm{SEM} ; \mathrm{n}=6 . * \mathrm{P}<0.01$ vs. controls.

To assess which of these two possibilities is more likely, we measured P62 protein expression and autophagic flux using the lysosomal inhibitor chloroquine $(50 \mu \mathrm{M})$. Figures $2 \mathrm{G}$ and $2 \mathrm{H}$ show that tempol increased $\mathrm{P} 62$ protein expression in $\mathrm{H} 9 \mathrm{C} 2$ cardiomyocytes under a basal condition. The autophagic flux was further determined by calculating the difference between absolute LC3 BII protein levels measured in the presence and absence of chloroquine in $\mathrm{H} 9 \mathrm{C} 2$ cardiomyocytes preincubated with vehicle, NAC or tempol. Under a basal condition, in NAC preincubated $\mathrm{H} 9 \mathrm{C} 2$ cardiomyocytes, autophagic flux was significantly lower than those measured in vehicle preincubated H9C2 cardiomyocytes (Figs 2I, 2J). Likewise, in tempol preincubated cardiomyocytes, autophagic flux under a basal condition was lower than those measured in vehicle preincubated H9C2 cardiomyocytes (Figs 2K, 2L). These results indicate that endogenous ROS promote myocyte autophagic flux under a basal condition.

Nutrient deprivation-induced ROS mediate autophagy in H9C2 cardiomyocytes

The nutrient deprivation has been shown to induce ROS (Sciarretta et al. 2013). To further determine the role of endogenous ROS in the regulation of autophagy under moderate stress conditions, H9C2 cardiomyocytes were exposed to the glucose- and serum-free medium with or without NAC or tempol treatment. Figures $3 \mathrm{~A}$ and $3 \mathrm{~B}$ show that the nutrient deprivation induces an increase in the LC3 II/I ratio, and the increase was preserved by NAC. Tempol treatment caused a further increase in the LC3 II/I ratio in H9C2 cardiomyocytes subjected to the nutrient deprivation (Figs 3C, 3D). 
A

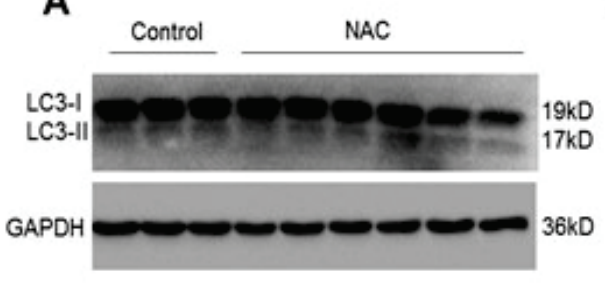

C
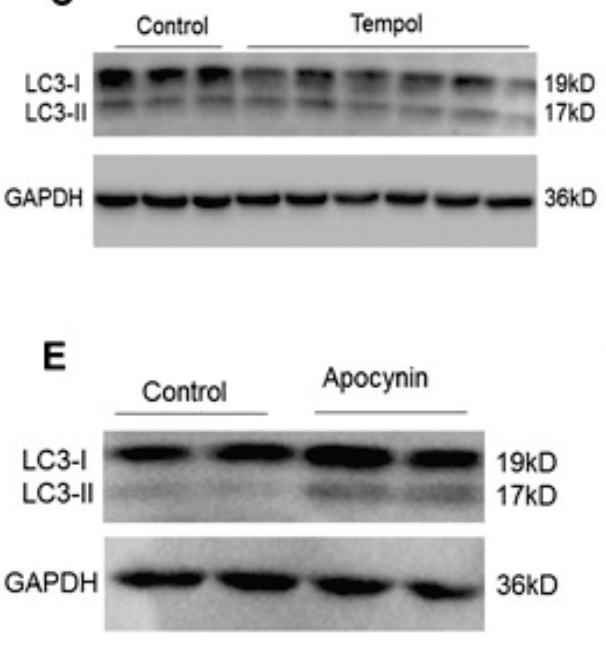

G

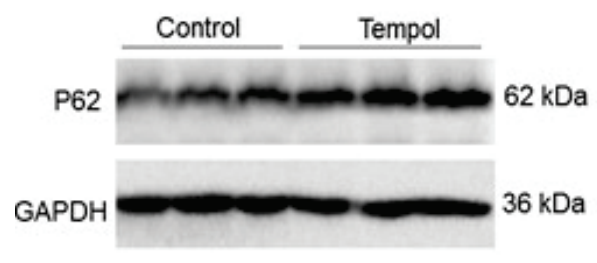

I

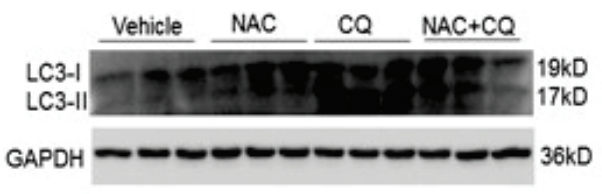

K

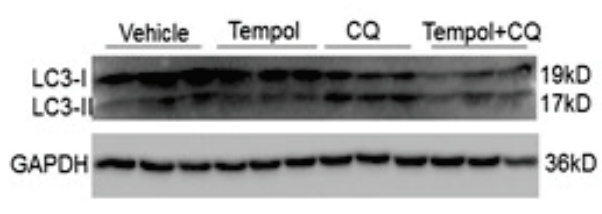

B

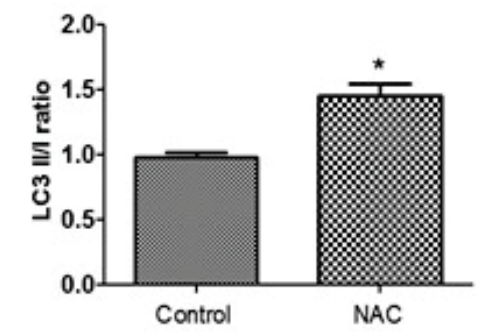

D
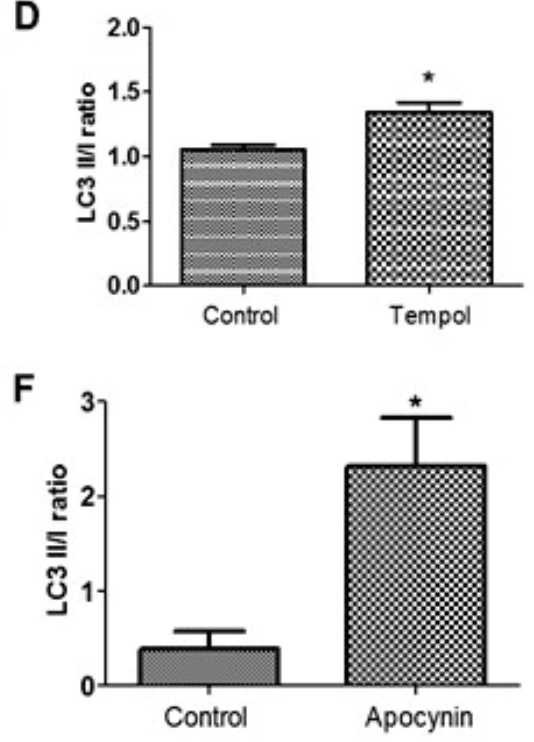

H
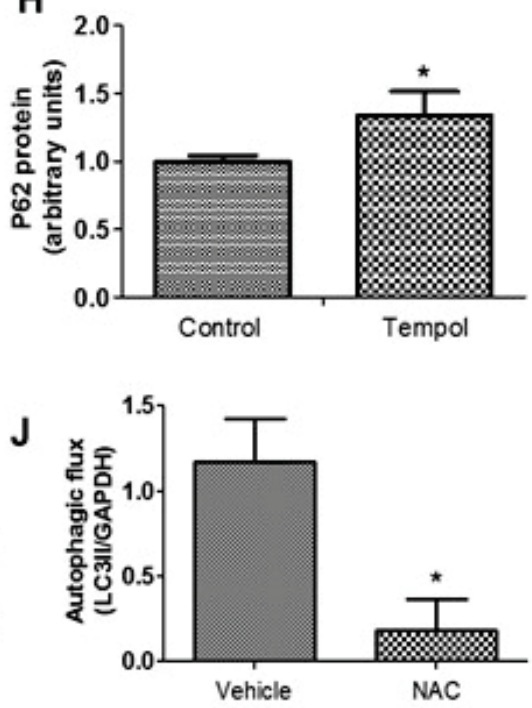

$\mathbf{L}$

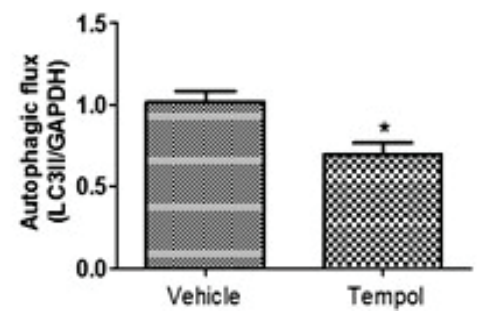

Fig. 2. Effects of antioxidants on autophagy in $\mathrm{H} 9 \mathrm{C} 2$ cardiomyocytes under a basal condition. (A) Representative Western blot of LC3 II and I proteins in $\mathrm{H} 9 \mathrm{C} 2$ cardiomyocytes treated with $\mathrm{N}$-acetyl-cysteine (NAC) or saline as a control for $6 \mathrm{~h}$. Equal loading of proteins is illustrated by GAPDH bands. (B) The ratio of LC3 II to LC3 I proteins. Values are means \pm SEM; $\mathrm{n}=6$. $* \mathrm{P}<0.001$ vs. control. (C) Representative Western blot of LC3 II and I proteins in $\mathrm{H} 9 \mathrm{C} 2$ cardiomyocytes treated with tempol or saline as a control for $6 \mathrm{~h}$. Equal loading of proteins is illustrated by GAPDH bands. (D) The ratio of LC3 II to LC3 I proteins. Values are means $\pm \mathrm{SEM}$; $\mathrm{n}=6 . * \mathrm{P}<0.001$ vs. control. (E) Representative Western blot of LC3 II and I proteins in $\mathrm{H} 9 \mathrm{C} 2$ cardiomyocytes treated with NADPH oxidase inhibitor apocynin or saline as a control for $6 \mathrm{~h}$. Equal loading of proteins is illustrated by GAPDH bands. (F) The ratio of LC3 II to LC3 I proteins. Values are means $\pm \mathrm{SEM} ; \mathrm{n}=6 . \quad * \mathrm{P}<0.001 \quad$ vs. control. (G) Representative Western blot of $\mathrm{P} 62$ protein in $\mathrm{H} 9 \mathrm{C} 2$ cardiomyocytes treated with tempol or saline as a control for $6 \mathrm{~h}$. Equal loading of proteins is illustrated by GAPDH bands. (H) Respective group densitometry analysis. Values are means $\pm \mathrm{SEM}$; $\mathrm{n}=6$. $* \mathrm{P}<0.05$ vs. controls. (I) Representative Western blot of LC3 II and I proteins in $\mathrm{H} 9 \mathrm{C} 2$ cardiomyocytes treated with vehicle, NAC, chloroquine (CQ) and NAC plus CQ for $6 \mathrm{~h}$. Equal loading of proteins is illustrated by GAPDH bands. (J) LC3 II autophagic flux in $\mathrm{H} 9 \mathrm{C} 2$ cardiomyocytes treated with vehicle or NAC. Values are means $\pm \mathrm{SEM} ; \mathrm{n}=6 . \quad * \mathrm{P}<0.001 \quad \mathrm{vs}$. vehicle. (K) Representative Western blot of LC3 II and I proteins in H9C2 cardiomyocytes treated with vehicle, tempol, chloroquine (CQ) and tempol plus CQ for $6 \mathrm{~h}$. Equal loading of proteins is illustrated by GAPDH bands. (L) LC3 II autophagic flux in $\mathrm{H} 9 \mathrm{C2}$ cardiomyocytes treated with vehicle or tempol. Values are means $\pm S E M ; n=6$. $* P<0.001$ vs. vehicle.
To examine the effects of nutrient deprivationinduced ROS on autophagic flux, H9C2 cardiomyocytes were exposed to the glucose- and serum-free medium, and treated with vehicle, chloroquine $(50 \mu \mathrm{M})$, NAC or chloroquine plus NAC for $6 \mathrm{~h}$. Figures $3 \mathrm{E}$ and $3 \mathrm{~F}$ show autophagic flux was significantly lower in the 
NAC treated group compared with those measured in the vehicle treated group. These findings suggest that moderate stress-induced ROS promote autophagic flux in H9C2 cardiomyocytes.

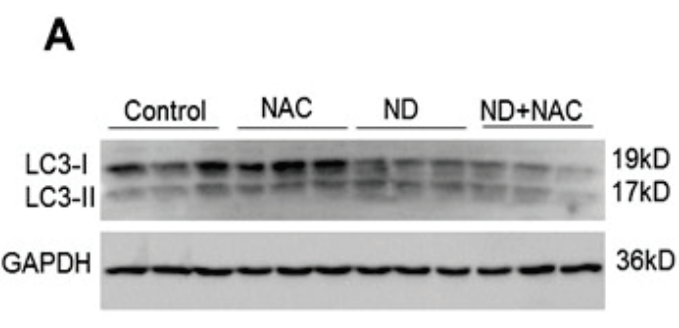

B

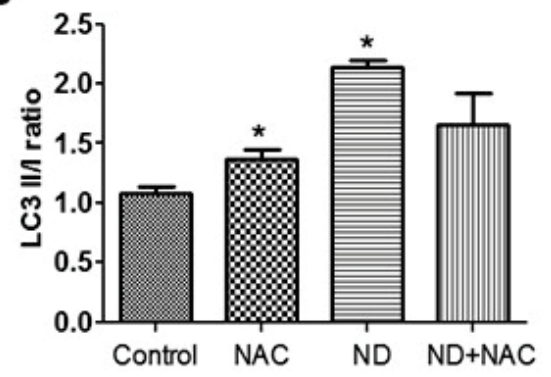

C

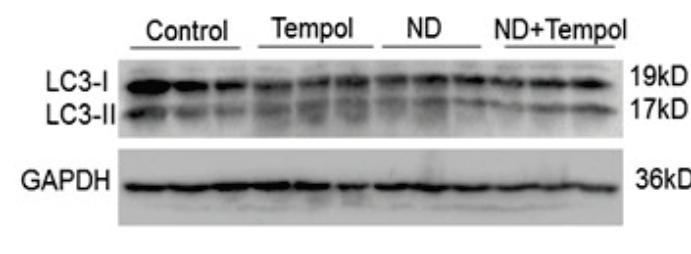

D

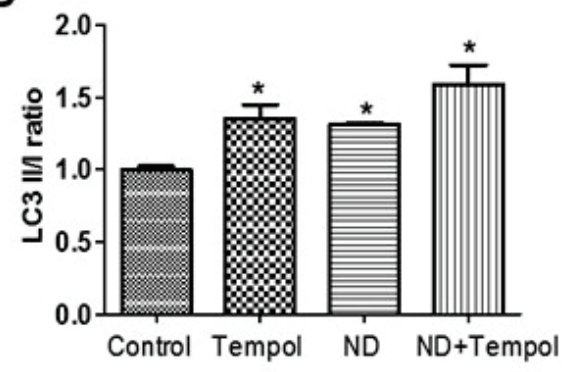

E

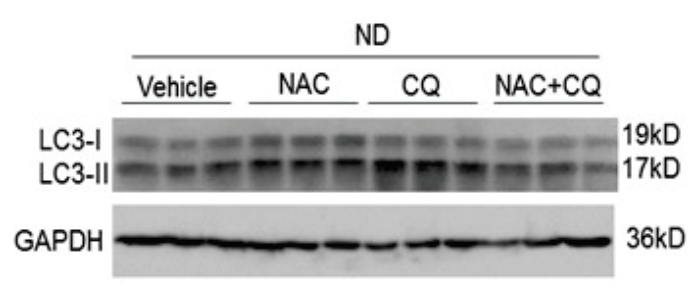

$\mathbf{F}$

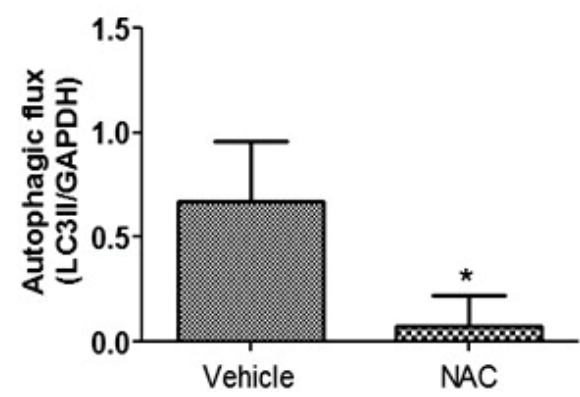

Fig. 3. Effects of antioxidants on autophagy in $\mathrm{H} 9 \mathrm{C} 2$ cardiomyocytes under a nutrient deprivation (ND) condition. (A) Representative Western blot of LC3 II and I proteins in H9C2 cardiomyocytes in DMEM containing saline as a control or N-acetyl-cysteine (NAC) and in ND containing saline or NAC for $6 \mathrm{~h}$. Equal loading of proteins is illustrated by GAPDH bands. (B) The ratio of LC3 II to LC3 I proteins. Values are means $\pm \mathrm{SEM} ; \mathrm{n}=6 . * \mathrm{P}<0.01$ vs. control. (C) Representative Western blot of LC3 II and I proteins in H9C2 cardiomyocytes in DMEM containing saline as a control or tempol and in ND containing saline or tempol for $6 \mathrm{~h}$. Equal loading of proteins is illustrated by GAPDH bands. (D) The ratio of LC3 II to LC3 I proteins. Values are means \pm SEM; $n=6 . * P<0.01$ vs. control. (E) Representative Western blot of LC3 II and I proteins in H9C2 cardiomyocytes in ND treated with vehicle, NAC, chloroquine (CQ) and NAC plus CQ for $6 \mathrm{~h}$. Equal loading of proteins is illustrated by GAPDH bands. (F) LC3 II autophagic flux in H9C2 cardiomyocytes treated with vehicle or NAC. Values are means $\pm S E M ; n=6$. $* P<0.01$ vs. vehicle.

The role of endogenous ROS in myocyte autophagy in rats in vivo

The role of endogenous ROS in the regulation of basal myocyte autophagy in vivo was evaluated by administering NAC to rats and by measuring changes in the levels of LC3 I and LC3 II proteins and the ratio of LC3 II to LC I proteins in myocardium. NAC treatment significantly increased the level of LC3 II protein and the ratio of LC3 II to LC3 I proteins (Figs 4A, 4B).
The signal pathways of endogenous ROS in the regulation of myocyte autophagy

Autophagy is initiated under control of the energy sensor AMP activated protein kinase (AMPK) and the amino-acid sensor mammalian target of rapamycin complex I (mTOR) (Matsui et al. 2007, Xiao et al. 2015). Autophagy is inhibited by protein kinase B (Akt) activation (Hua et al. 2011). AMPK, mTOR and Akt are well known to be under the influence of ROS (Essick et 
al. 2013, Horie et al. 2008). To examine ROS regulation of p-AMPK and p-Akt proteins, healthy adult male rats were treated with antioxidant NAC under a basal condition. Compared with the vehicle treated group, in the NAC treated group, $\mathrm{p}$-AMPK protein had no change under the basal condition (data not shown), but p-Akt protein expression was significantly increased under the basal condition (Figs 4C, 4D).
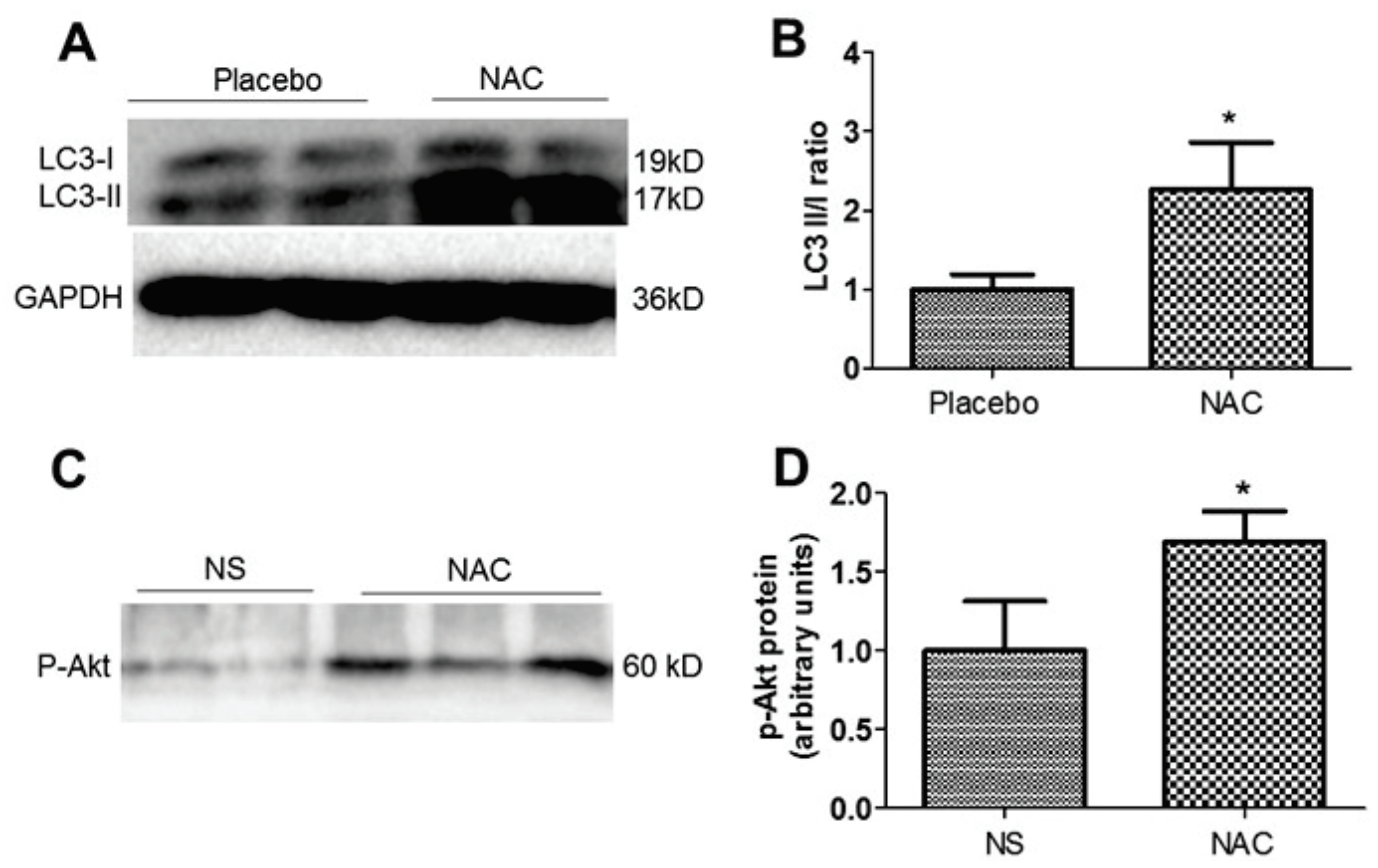

Fig. 4. Effects of antioxidant $\mathrm{N}$-acetyl-cysteine (NAC) on autophagy and Akt protein in myocardium in rats. (A) Representative Western blot of LC3 II and I proteins in rats treated with NAC or placebo for 2 weeks. Equal loading of proteins is illustrated by GAPDH bands. (B) The ratio of LC3 II to LC3 I proteins. Values are means \pm SEM; $n=6 . * P<0.001$ vs. placebo. (C) Representative Western blot of p-Akt protein in rats treated with NAC or normal saline (NS) for 2 weeks. (D) Respective group densitometry analysis. Values are means $\pm \mathrm{SEM} ; \mathrm{n}=4 .{ }^{*} \mathrm{P}<0.05$ vs. NS.

\section{Discussion}

In the present study, we have demonstrated that (1) in cultured $\mathrm{H} 9 \mathrm{C} 2$ cardiomyocytes, under a basal condition, antioxidant NAC or tempol and NADPH oxidase inhibitor apocynin induced an increase in the ratio of LC3 II/I proteins; tempol increased P62 protein expression; NAC or tempol reduced LC3 II autophagic flux; (2) under a nutrient deprivation condition, the LC3 II/I ratio was increased and the increase was preserved by NAC and promoted by tempol; under this condition, NAC also reduced LC3 II autophagic flux; (3) In rats in vivo, NAC treatment caused an increase in the LC3 II/I ratio in myocardium; (4) NAC treatment markedly increased p-Akt protein expression. These results suggest that endogenous ROS promote autophagy flux under physiological conditions, and this effect was mediated, at least in part, through Akt inhibition.

Autophagy has been shown to increase during myocardial ischemia and reperfusion and heart failure (Nakai et al. 2007, Zhu et al. 2007), which are associated with increased oxidative stress (Burgoyne et al. 2012). In adult rat ventricular cardiomyocytes in culture, high concentrations of $\mathrm{H}_{2} \mathrm{O}_{2}$ increase autophagy (Hariharan et al. 2011, Essick et al. 2013). In H9C2 cardiomyocytes, high concentrations of $\mathrm{H}_{2} \mathrm{O}_{2}$ also induce autophagy (Hsu et al. 2014). Our present study further confirmed these results showing that $\mathrm{H}_{2} \mathrm{O}_{2}$ induces autophagy as evidenced by increases in the ratio of LC3 II/I proteins and Atg5 protein expression in $\mathrm{H} 9 \mathrm{C} 2$ cardiomyocytes at a dose-dependent manner. These findings suggest that exogenous ROS mediate myocyte autophagy.

Autophagy is either positively or negatively regulated by oxidative stress (Kubli and Gustafsson 2012). However, there is no report about the role of endogenous ROS in myocyte autophagy. In the present study, we have found that antioxidant NAC or tempol increased the LC3 II/I ratio in cardiomyocyte in vitro and in myocardium in rats in vivo under a basal physiological condition. These results indicate that antioxidants trigger the accumulation of autophagosomes, which results from either increased synthesis or decreased degradation. 
P62, an adaptor protein that links aggregated proteins in autophagosomes and is degraded in autolysosomes, has been used to indicate autophagic flux (Matsui et al. 2007). We have found that tempo increased P62 protein expression in cardiomyocytes under a basal condition, indicating impaired autophagic flux. We have further found that NAC or tempol inhibited autophagic flux under the basal condition evaluated by the lysosomal inhibitor chloroquine. These findings suggest that endogenous ROS promote autophagic flux in cardiac myocytes under the basal physiological condition. These findings are supported by the previous studies showing that overexpression of catalase that detoxifies $\mathrm{H}_{2} \mathrm{O}_{2}$ tends to increase myocardial LC3 II/I although this study did not reach statistical significance and did not show the effect of catalase on autophagic flux (Turdi et al. 2012). In agreement with the present study, endogenous ROS has been shown to promote autophagic flux in skeletal muscle cells (Rahman et al. 2014). In addition, we have found that NADPH oxidase inhibitor apocynin increased the LC3 II/I ratio under a basal physiological condition, suggesting that NADPH oxidase may be a major source of endogenous ROS which regulate myocyte autophagy.

The levels of intracellular ROS increase in response to nutrient deprivation (Scherz-Shouval et al. 2007). In the present study, we have found that nutrient deprivation increased the ratio of LC3 II/I proteins and the increase was preserved by NAC or enhanced by tempol treatment. We have further demonstrated that NAC or tempol treatment reduced autophagic flux. These results indicate that endogenous ROS induce autophagic flux under the nutrient deprivation condition. These findings are in consistent with the previous reports showing that starvation induces autophagic flux (Hariharan et al. 2011). The notion is also supported by other report demonstrating that nutrient deprivationderived ROS induce autophagic flux in skeletal muscle (Rahman et al. 2014). Under glucose deprivation conditions, a study from Sadoshima's group has shown that NADPH oxidase subunit Nox4 promotes myocyte autophagy, suggesting that Nox4 mediates autophagy in response to energy stress in myocytes (Sciarretta et al. 2013).

Studies have shown that AMPK/mTOR, Akt and Beclin1 are involved in the regulation of myocyte autophagy (Matsui et al. 2007). AMPK $\alpha$ activation has been shown to inhibit mTOR activation and the inhibition of mTOR induces myocyte autophagy (Matsui et al. 2007, Xiao et al. 2015). In the present study, we have demonstrated that under the basal condition, NAC markedly increased Akt protein expression. These results indicate that endogenous ROS may inhibit Akt activity. Since Akt is an inhibitor of autophagy, these findings suggest that endogenous ROS promote myocyte autophagy probably through Akt inhibition.

The limitation of the present study is that we examined the role of endogenous ROS in myocyte autophagy using antioxidants. Further study is warranted to investigate the role of specific ROS such as superoxide and $\mathrm{H}_{2} \mathrm{O}_{2}$ in myocyte autophagy in cultured myocyte and transgenic animals. It is important to further elucidate the role of endogenous ROS-mediated autophagy on cell viability, metabolism and function.

In summary, antioxidant NAC or tempol decreased autophagic flux in cultured cardiomyocytes under the basal or nutrient deprivation conditions. NAC increased p-Akt protein expression. These findings suggest that endogenous ROS promote autophagy flux under physiological conditions, and this effect is mediated, at least in part, through Akt inhibition.

\section{Conflict of Interest}

There is no conflict of interest.

\section{Acknowledgements}

This study was supported in part by the National Natural Science Foundation of China (81270307) (81470526) (81670369) and the Shanxi 100 Talent Plan Research Fund (145451529913).

\section{References}

BURGOYNE JR, MONGUE-DIN H, EATON P, SHAH AM: Redox signaling in cardiac physiology and pathology. Circ Res 111: 1091-1106, 2012.

ESSICK EE, WILSON RM, PIMENTEL DR, SHIMANO M, BAID S, OUCHI N, SAM F: Adiponectin modulates oxidative stress-induced autophagy in cardiomyocytes. PLoS One 8: e68697, 2013.

GRIEVE DJ, BYRNE JA, SIVA A, LAYLAND J, JOHAR S, CAVE AC, SHAH AM: Involvement of the nicotinamide adenosine dinucleotide phosphate oxidase isoform Nox2 in cardiac contractile dysfunction occurring in response to pressure overload. J Am Coll Cardiol 47: 817-826, 2006. 
HARIHARAN N, ZHAI P, SADOSHIMA J: Oxidative stress stimulates autophagic flux during ischemia/reperfusion. Antioxid Redox Signal 14: 2179-2190, 2011.

HORIE T, ONO K, NAGAO K, NISHI H, KINOSHITA M, KAWAMURA T, WADA H, SHIMATSU A, KITA T, HASEGAWA K: Oxidative stress induces GLUT4 translocation by activation of PI3-K/Akt and dual AMPK kinase in cardiac myocytes. J Cell Physiol 215: 733-742, 2008.

HSU HC, CHEN CY, CHIANG CH, CHEN MF: Eicosapentaenoic acid attenuated oxidative stress-induced cardiomyoblast apoptosis by activating adaptive autophagy. Eur J Nutr 53: 541-547, 2014.

HUA Y, ZHANG Y, CEYLAN-ISIK AF, WOLD LE, NUNN JM, REN J: Chronic Akt activation accentuates aginginduced cardiac hypertrophy and myocardial contractile dysfunction: role of autophagy. Basic Res Cardiol 106: 1173-1191, 2011.

KANAMORI H, TAKEMURA G, GOTO K, MARUYAMA R, TSUJIMOTO A, OGINO A, TAKEYAMA T, KAWAGUCHI T, WATANABE T, FUJIWARA T, FUJIWARA H, SEISHIMA M, MINATOGUCHI S: The role of autophagy emerging in postinfarction cardiac remodelling. Cardiovasc Res 91: 330-339, 2011.

KUBLI DA, GUSTAFSSON AB: Mitochondria and mitophagy: the yin and yang of cell death control. Circ Res 111: 1208-1221, 2012.

LAVANDERO S, TRONCOSO R, ROTHERMEL BA, MARTINET W, SADOSHIMA J, HILL JA: Cardiovascular autophagy: concepts, controversies, and perspectives. Autophagy 9: 1455-1466, 2013.

LI B, TIAN J, SUN Y, XU TR, CHI RF, ZHANG XL, HU XL, ZHANG YA, QIN FZ, ZHANG WF: Activation of NADPH oxidase mediates increased endoplasmic reticulum stress and left ventricular remodeling after myocardial infarction in rabbits. Biochim Biophys Acta 1852: 805-815, 2015.

MARAMBIO P, TORO B, SANHUEZA C, TRONCOSO R, PARRA V, VERDEJO H, GARCIA L, QUIROGA C, MUNAFO D, DIAZ-ELIZONDO J, ET AL.: Glucose deprivation causes oxidative stress and stimulates aggresome formation and autophagy in cultured cardiac myocytes. Biochim Biophys Acta 1802: 509-518, 2010.

MATSUI Y, TAKAGI H, QU X, ABDELLATIF M, SAKODA H, ASANO T, LEVINE B, SADOSHIMA J: Distinct roles of autophagy in the heart during ischemia and reperfusion: roles of AMP-activated protein kinase and Beclin 1 in mediating autophagy. Circ Res 100: 914-922, 2007.

NAKAI A, YAMAGUCHI O, TAKEDA T, HIGUCHI Y, HIKOSO S, TANIIKE M, OMIYA S, MIZOTE I, MATSUMURA Y, ASAHI M, ET AL.: The role of autophagy in cardiomyocytes in the basal state and in response to hemodynamic stress. Nat Med 13: 619-624, 2007.

PENG YW, BULLER CL, CHARPIE JR: Impact of N-acetylcysteine on neonatal cardiomyocyte ischemia-reperfusion injury. Pediatr Res 70: 61-66, 2011.

QIN F, SIMEONE M, PATEL R: Inhibition of NADPH oxidase reduces myocardial oxidative stress and apoptosis and improves cardiac function in heart failure after myocardial infarction. Free Radic Biol Med 43: 271-281, 2007.

RAHMAN M, MOFARRAHI M, KRISTOF AS, NKENGFAC B, HAREL S, HUSSAIN SN: Reactive oxygen species regulation of autophagy in skeletal muscles. Antioxid Redox Signal 20: 443-459, 2014.

SANTOS CX, RAZA S, SHAH AM: Redox signaling in the cardiomyocyte: From physiology to failure. Int J Biochem Cell Biol 74: 145-151, 2016.

SCHERZ-SHOUVAL R, SHVETS E, FASS E, SHORER H, GIL L, ELAZAR Z: Reactive oxygen species are essential for autophagy and specifically regulate the activity of Atg4. EMBO J 26: 1749-1760, 2007.

SCIARRETTA S, ZHAI P, SHAO D, ZABLOCKI D, NAGARAJAN N, TERADA LS, VOLPE M, SADOSHIMA J: Activation of NADPH oxidase 4 in the endoplasmic reticulum promotes cardiomyocyte autophagy and survival during energy stress through the protein kinase RNA-activated-like endoplasmic reticulum kinase/eukaryotic initiation factor 2alpha/activating transcription factor 4 pathway. Circ Res 113: 1253-1264, 2013.

TURDI S, HAN X, HUFF AF, ROE ND, HU N, GAO F, REN J: Cardiac-specific overexpression of catalase attenuates lipopolysaccharide-induced myocardial contractile dysfunction: role of autophagy. Free Radic Biol Med 53: 1327-1338, 2012. 
XIAO Q, YANG Y, QIN Y, HE YH, CHEN KX, ZHU JW, ZHANG GP, LUO JD: AMP-activated protein kinasedependent autophagy mediated the protective effect of sonic hedgehog pathway on oxygen glucose deprivation-induced injury of cardiomyocytes. Biochem Biophys Res Commun 457: 419-425, 2015.

ZHU H, TANNOUS P, JOHNSTONE JL, KONG Y, SHELTON JM, RICHARDSON JA, LE V, LEVINE B, ROTHERMEL BA, HILL JA: Cardiac autophagy is a maladaptive response to hemodynamic stress. $J$ Clin Invest 117: 1782-1793, 2007. 